\title{
Rehabilitation and Discharge
}

\author{
Silvia Barberi and Lucia Mielli
}

Fragility fracture is the result of a combination of bone fragility and falls and often leads to hip fracture, a devastating injury for both the patient and their family. The recovery process requires both physical and psychosocial care [1], and much research has focused on physical function, including post-hospital discharge and rehabilitation. All patients presenting with a fragility fracture following a fall should have a full multidisciplinary assessment and interventions to prevent future fractures and falls. The care, rehabilitation and discharge of patients with hip fracture are a significant challenge for many services, but the quality and cost-effectiveness of such care vary considerably. This chapter aims to discuss the role of the nurse in the rehabilitation and hospital discharge phases of the care trajectory.

\subsection{Learning Outcomes}

At the end of this chapter, and following further study, the nurse will be able to:

- Describe strategies for effective rehabilitation and post-hospital care of older people following fragility fracture

- Describe the role of the nurse in rehabilitation

- Identify and apply strategies for supporting patient motivation and increase selfmanagement during recovery

- Effectively plan patient discharge with the involvement of the patient, family and caregivers.

S. Barberi $(\bowtie)$

Azienda Ospedaliera San Giovanni Addolorata, Rome, RM, Italy

L. Mielli

Azienda Sanitaria Unica Regionale (ASUR) Marche, Ancona, AN, Italy

(C) The Editor(s) (if applicable) and the Author(s) 2018 


\subsection{Rehabilitation}

Following fragility fracture, and especially hip fracture, patients have complex medical, surgical and rehabilitation needs, and social and psychological factors such as fear of falling, self-efficacy, perceived control and coping strategies are important factors in recovery and rehabilitation. Care and rehabilitation of patients following hip fracture are particularly challenging for trauma services, but units that are able to provide good care for these patients will also be able to provide effective care for the complete range of other fragility fractures encountered. The multifactorial nature of the problems facing fragility fracture patients requires a multidisciplinary approach with an emphasis on effective teamwork along with close collaboration between the medical, nursing, physiotherapy, occupational therapy and social care teams. Good multidisciplinary working requires positive attitudes, good communication and sharing of information, an adaptive and flexible approach to collaboration and deep commitment from all concerned to promote quality care and good outcomes for patients.

The primary outcomes of rehabilitation are independence in physical function and quality of life. Poor outcomes of recovery and rehabilitation amount to failure to return to independent living and readmission to hospital. Effective rehabilitation is important in promoting independence and in enabling the patient to reach their potential and return home, as well as minimising costs by reducing the length of hospital stay [2], although there is limited evidence relating to how different care strategies impact on rehabilitation and discharge [3] and there is limited information about who can best provide this care. Early supported multidisciplinary rehabilitation can reduce hospital stay, improve early return to function and impact positively on both readmission rates and the level of public-funded nursing care required.

Increasingly, community rehabilitation schemes are being developed that facilitate early discharge of less frail fracture patients to their own home from the orthopaedic ward. Earlier discharge can be facilitated by referral to a community rehabilitation team, day hospital or other community-based rehabilitation service. Such ongoing rehabilitation allows patients to continue to improve functionally and progress towards their goal after leaving hospital. Collaborative approaches in the acute setting, such as hip fracture programmes [4], can be effective in improving outcomes, and patient rehabilitation and discharge can benefit from intermediate care initiatives such as early supported discharge and care pathways.

\subsubsection{Rehabilitation Pathways}

From admission, patients should be offered a formal, acute, orthogeriatric or orthopaedic ward-based hip fracture programme that includes all of the following:

- Comprehensive multidisciplinary geriatric/orthogeriatric assessment and continuous review

- Rapid preoperative optimisation of fitness for surgery

- Early identification of individual goals for rehabilitation, to recover mobility and independence and to facilitate return to pre-fracture residence and long-term wellbeing 
- Liaison or integration with related services, particularly mental health, falls prevention, bone health, primary care and social services

- Clinical and service governance responsibility for all stages of the pathway of care and rehabilitation, including those aspects delivered in the community.

In many localities, nurses in specialist and advanced roles identify patients suitable for enhanced rehabilitation schemes and ensure their smooth passage through the perioperative period into the rehabilitation phase. After immediate post-operative recovery, it is essential that patients follow a rehabilitation pathway that includes six main elements [3]:

- Assessment of frailty (Chap. 2)

- Establishing goals to maximise mobility and other aspects of function; therapy provided by physiotherapists and occupational therapists has the potential to accelerate the recovery of mobility; the timing of physiotherapy assessment and intervention is important and should begin within $48 \mathrm{~h}$ of surgery

- Providing occupational therapy services to assess the need for aids

- Determining strategies to support and improve independence in activities of daily living

- Medication management to ensure all prescribed medications are necessary, the use of antipsychotics and sedatives is minimised and there is effective pain management

- Secondary fracture and fall prevention (Chap. 3).

After hip fracture surgery, patients follow individual pathways through recovery and rehabilitation but, at the same time, patients often experience a common trajectory. "Integrated care pathways" (ICPs) were developed as a way to standardise treatment protocols following hip fracture as well as individualise multidisciplinary management and rehabilitation. These are structured multidisciplinary care plans that describe in detail each step in the process towards rehabilitation and discharge and act as the patient's care record, with the aim of ensuring that the patient receives the recommended standards of care at the appropriate time [5]. Some units have found ICPs helpful in improving key areas of hip fracture care such as optimisation for surgery (often now enshrined in enhanced recovery programmes), early mobilisation, communication with the patient and family, rehabilitation and discharge planning. If such approaches are to be successful as catalysts for high-quality care and rehabilitation, it is essential that all members of the MDT are involved in their development and continuing use.

\subsubsection{Mobility and Exercise}

As discussed in Chap. 6, mobility and exercise are central to recovery and rehabilitation from injury and surgery. Supported mobility should be an integral part of nursing, using everyday activities such as getting out of bed and walking to the toilet as part of the rehabilitation programme. It is important that this process begins as early as possible after surgery as there is a statistically and clinically significant 
increase in independence for patients who have early mobilisation compared to delayed mobilisation [6]. Sherrington et al. [7] suggest that exercise programmes should last for at least $2 \mathrm{~h}$ a week, for at least 6 months, to reach the desired outcomes.

Mobilisation and other activities need to be reintroduced into patients' activity gradually as their physical recovery from surgery progresses. Important aims include the ability to transfer independently, for example, between a bed and a chair, or ability to rise from a chair [4] as well as walking. When engaged in regularly, these activities can help to improve muscle strength and can impact positively on falls, length of stay, discharge destination, independence in ADLs such as washing and bathing, as well as more complex tasks such as meal preparation. Although this activity should be under the supervision of therapists, it is the nursing team who will most often supervise the patient. Tailored exercises to help improve muscle strength and function should be prescribed by the physiotherapist but supervised by the nursing team and should begin while the patient is in hospital but continued after discharge.

\subsubsection{Patient Motivation}

The process of rehabilitation involves a transition from one state (helplessness) to another (independence) that needs significant and sustained effort by patients [5]. This process is often described by those who have experienced it as a very difficult event that is fraught with uncertainty, passivity and declining function, needing support in using their inner resources, while they are striving to regain function and independence [8]. To be successful in rehabilitation goals, older people need to be motivated to concord with therapy and exercise programmes and other activities. Social and psychological factors such as fear of falling, selfefficacy and coping strategies are thought to be important in the recovery from hip fracture in older people [9]. Improvements in patient motivation can be achieved by developing a constantly positive approach to patients, with an encouraging attitude and empowering patients to become actively involved in their rehabilitation [10].

Rehabilitation involves major effort from patients. Patient views about their hip fracture and its management and the way they are provided with information are important elements of the natural recovery and treatment process. Caregivers also need information and can influence the recovery process. Patients should receive information and education about the fracture, surgery, risk of future fractures, follow-up and duration of therapy as well as the rehabilitation process. Timely and clear information can reduce stress and uncertainty for patients and potentially improve their outcomes. Caregivers also play a key role in the rehabilitation of elderly patient with hip fracture and can help with motivation, training for walking and facilitation of access to health services, among other aspects. Participation in decision-making, improved access to legal and medical information, possibility of sharing care experiences, presence of a secondary caregiver and increased social 
support improve the self-efficacy of care, but caregiver overload needs to be carefully considered.

\subsection{Discharge and Post-hospital Care}

The discharge of an older person from an acute hospital to the most appropriate setting following hip fracture surgery is a complex undertaking requiring careful planning and, if ineffectively conducted, can be the weak link in the passage of the patient from one care setting to another. Premature discharge or discharge to an unsuitable environment can result in hospital readmission. Early hospital discharge may not lead to overall cost-savings if it results in the need for more intense subsequent health-care utilisation, such as ED visits or rehospitalisation. Hospital readmissions are often the result of a fragmented health and social care system [11] and increasing evidence indicates that patients are particularly vulnerable and more likely to experience negative outcomes during these hospital readmissions [12].

Many factors that can increase the likelihood of readmission can be modified so need be considered in the way services are designed and developed. Such factors include; premature discharge, inadequate post-discharge support, insufficient followup, therapeutic errors including adverse drug events and other medication-related issues, inadequate transfer handovers and complications of hospital procedures and surgery such as nosocomial infections, pressure ulcers and patient falls.

The patient and family have a right to be involved and supported at every stage of the process so collaboration and continuity of care are central. It is essential that the older person and their family are adequately prepared for discharge, that a care pathway is activated that continues following discharge and that the role of the family and informal carers as partners in the care team is facilitated [13]. Development of a discharge plan must begin as early as possible during the hospital stay, to ensure that patient education and support are provided to facilitate independence and so that the patient can develop an understanding of their health condition and acquire the knowledge and skills needed to self-care independently or with caregiver/family support.

Patients leaving hospital following hip fracture surgery always need further care. To enable discharge, the health-care team must determine the most appropriate setting for ongoing care, considering the continuing care, medical, functional and social needs, and decision-making capacity of the patient. The MDT should collaborate with the patient/family/caregivers and other stakeholders to determine the most suitable plan. Several factors must be considered including; cognitive status, activity level and functional capacity, current home suitability, availability of informal and formal care, availability of transportation and availability of services for ongoing care. The severity of functional impairments and the need for assistance with activities of daily living (ADLs) often determine whether a patient can be safely managed at home or requires care at a skilled nursing facility ("nursing home") or extended care facility ("residential home") with attention to the need for supervision of ADLs and safety awareness. 


\subsubsection{Discharge Home}

For successful discharge home, patients (with help from family or other caregivers, if available) should be able, as a minimum, to:

- Obtain and self-administer medications

- Perform self-care activities

- Eat an appropriate diet or otherwise manage nutritional needs

- Engage with follow-up care.

Availability of appropriate services in the community can influence whether the patient may be safely discharged home. Home services may allow patients who would otherwise need residential care to manage their care needs at home. The lack of a system that ensures continuity of care following discharge home, or other location, can cause serious errors including adverse medication events [14].

\subsubsection{Discharge to Another Setting}

If discharge home is not appropriate, transfer to another inpatient or residential facility for ongoing care must be arranged. Determining the most appropriate setting for ongoing care involves assessing and matching needs with the capabilities of the potential care setting. One model to help accomplish this involves assessing a set of parameters that describe generic clinical characteristics (medical and surgical issues, mental and emotional status, physical functioning and environment) that are largely independent of the patient's specific diagnosis. These needs are then matched with the services offered at different types of facilities. Once the care team, patient and family have decided that discharge to an alternate facility is necessary, referrals can be made to facilities that are appropriate and meet the patient and family desires and the patient can be screened for acceptance.

Three main types of care facility exist depending on the locality/region/country, each with a different function:

- Acute care hospitals

- Inpatient rehabilitation hospitals, intermediate care/step down units and longterm acute care hospitals

- Nursing and residential care facilities (private or government funded).

Poor information transfer from hospital-based providers to other facilities is common and can contribute to poor discharge/transfer outcomes including the need for readmission, temporary or permanent disability or even death. Discharge information, both written and verbal, should be reviewed with the patient/family and caregivers with an emphasis on assessing and ensuring comprehension. At discharge, the patient should be provided with a document that includes language and literacy-appropriate instructions and patient education materials to help in 
successful transition from the hospital. These documents should be brief, focused on critical information for the patient and focused on what the patient needs to understand to manage after discharge. One model for patient materials, developed by the National Patient Safety Foundation [15], called "Ask Me 3", includes the following information:

1. What is my main problem? (Why was I in the hospital?)

2. What do I need to do? (How do I manage at home and what should I do if I run into problems?)

3. Why is it important for me to do this?

\subsubsection{The Discharge Process}

A critical issue leading to discharge problems is lack of planning of the discharge itself. The discharge process must begin on admission to allow time and resources for discharge planning. There are three phases that characterise the discharge process: (1) admission, (2) hospitalisation and (3) discharge.

The admission phase: Within $48 \mathrm{~h}$ of admission, the Blaylock Risk Assessment Screening Score (BRASS), a tool that can be used to identify patients who may require a more comprehensive discharge plan, can be used to identify patients at risk of difficult discharge [16] and a referral can be made to the discharge liaison service.

The hospitalisation phase: Once a place of discharge has been decided, contact can be made. If discharge is to be to a continuing care facility (rehabilitation/intermediate care unit or nursing/residential home), individuals involved in the admission to the care facility should visit the patient to assess their suitability for the facility and to discuss this with the patient and family. This visit can enable community care professionals or a continuing care manager to undertake a detailed assessment of the patient's function and need for continuing care interventions. This can be done using a specific measure of function such as the FIM ${ }^{\circledR}$ (Functional Independence Measure), an international standard for the measurement of disability; using cumulative scores produces a quantitative index of the person's function. The FIM ${ }^{\mathrm{TM}}$ score has proven validity as an index of rehabilitation efficacy and can be used in acute hospitals, post-acute rehabilitation hospitalisation, nursing homes and home care.

An initial individualised care plan should be developed based on the person's overall condition and function (degree of pre- and post fracture autonomy, comorbidity, polypharmacotherapy, postfracture conditions, delirium and recovery motivation) and point in the post-hospital trajectory (intensive, extensive rehabilitation). If discharge home is planned, requests for appropriate aids can be made at this time. The education and training of patients and informal caregivers should also begin as soon as possible and continued once the patient is home.

The discharge phase: The multidisciplinary team collaborates to devise and operationalise a definitive individualised care plan for discharge. Assessment of the degree of independence and autonomy achieved by the patient during their hospital 
stay and recovery and their readiness for discharge can be assessed. Important arrangements for transport, follow-up, equipment and drugs should also be made by the discharge coordinator. Ultimately, verification of the patient's arrival home and that services have commenced will complete the process. If home care is being provided, care will be formally transferred to the health professional who is assigned to the patient and family, the coordinator of the rehabilitation facility or the care leader in the residential care facility.

\subsubsection{Continuity of Care}

Continuity of care has three aims; best quality of care, the best health outcomes for the patient and cost reduction [17], and is achieved through:

1. The transfer of information and sharing of the patient's story with other professionals

2. Timely collection of information and activation of necessary resources immediately following discharge

3. Effective discharge planning

4. Monitoring and accompaniment

5. The assessment of needs of the use and caregivers.

Continuity of care can be achieved by one health or social care professional taking responsibility for the transition between care settings and ensuring that effective care is provided throughout the transition while focusing on the person and their family [13]. Taking this responsibility ensures continuity of care from one operating unit to another and across different levels of the health and social care system. This also ensures that the complex care process is integrated and is led in a way that guarantees that the older person receives a coordinated set of interventions aimed at meeting their complex needs. This enables governance of a complex and integrated care process in its various stages and guarantees that a coordinated set of interventions aimed at satisfying complex needs is in place [18].

There are several critical issues that can lead to discharge problems:

- Lack of a planned discharge date

- A high level of support needed for family who are inadequately prepared for the discharge

- Delayed activation of community services

- Poor attention to the needs of frail older people

- Lack of intermediate care services

- Lack of residential care facilities

- Poor knowledge of formal and informal services and how to access them

- Difficulty dealing with the paperwork

- Lack of support and a sense of abandonment of caregivers [13]. 


\subsubsection{The Nurse's Role in Discharge}

Nurses have an important role in ensuring continuity of care between settings and can act as a coordinator, supporting hospital staff involved in the discharge process, reducing hospital readmissions, ensuring continuity of care and educating patients about safety in continued care [19]. Care that began in the acute hospital should be continued following discharge through specialist nurse-led care in the rehabilitation hospital, intermediate care, home care or residential care setting led by a nurse with masters' level gerontological education [9].

In many health systems, a nurse is employed as a case manager or discharge coordinator whose key role is to support discharge planning and negotiating the different parts of care services and formal and informal care networks, particularly during transfer from one service to another. This includes supporting the person who cannot directly, or through a family member, interface with the care networks themselves. The nurse case manager is responsible for [20]:

1. Care integration: ensuring coherence between what has been planned and what is achieved

2. Coordination of care: ensuring that the care plan is followed by all those involved in its provision

3. Continuity of care: ensuring the implementation of the plan of care across all areas of care.

Nurses are the most appropriate health professionals to act as case manager [21] and/or discharge coordinator because of:

- Their clinical skills

- Ability to improve the coordination of services

- They are more generalist than other professionals

- They excel in giving direct care and pay attention to the relationship between care quality and cost and the natural evolution of nursing care

- Their ability to understand the holistic needs of patients and their current and potential problems [20].

There are many skills needed by the nurse case manager including; agent of change, clinical knowledge, identification and assignment of cases, consultancy, educator, coordinator and facilitator of care, resource manager and outcome and quality manager and advocate.

\subsection{Summary of Key Points for Learning}

- Rehabilitation and discharge planning should begin as soon as possible after admission

- The primary outcomes of rehabilitation are independence in physical function and quality of life 
- Effective rehabilitation is important in promoting independence and in enabling the patient to reach their potential and return home

- Early supported multidisciplinary rehabilitation can reduce hospital stay, improve early return to function and impact positively on both readmission rates and the level of care required

- Supported exercise and mobility include interventions involving using everyday activities as part of the rehabilitation programme to increase independence

- The discharge of an older person from an acute hospital is complex and requires careful planning and, if ineffectively conducted, can affect patient outcomes

- Nurses ensure continuity of care between settings and can act as a coordinator in the discharge process.

\subsection{Recommended Further Study}

- Think about a patient whose rehabilitation you are currently or were recently involved in. Based on your learning from this chapter, identify areas in their care you could improve.

- Carefully read the following article and reflect on how you might apply it to your practice: Lindberg, L. et al. (2017) Changing caring behaviours in rehabilitation after a hip fracture-A tool for empowerment? Psychology Health and Medicine 22(6):663-672 https://doi.org/10.1080/13548506.2016.1211294.

- Examine the documentation for a patient in whose discharge you were recently involved - if possible, one for which you acted as discharge coordinator. Reflect on the quality of the discharge, bearing in mind what you have learnt from this chapter and consider how the discharge might have been improved by your actions. Identify an action plan for improving the way you approach patient discharge as a coordinator.

- Seek advice and mentorship from other expert clinicians such as physiotherapists, social workers and those who are experts in rehabilitation or discharge planning.

\subsection{How to Self-Assess Learning}

To identify learning achieved and the need for further study, the following strategies may be helpful:

- Examine local documentation of nursing care, regarding rehabilitation and discharge status, and use this to assess your knowledge and performance.

- Meet with specialists and other members of the team to keep up to date on new evidence and disseminate it to colleagues. The conversation in these meetings can include any recent new practices, guidance, knowledge or evidence. 


\section{References}

1. British Orthopaedic Association (BOA) (2007) The care of patients with fragility fracture. British Orthopaedic Association

2. Menzies IB et al (2010) Prevention and clinical Management of hip fractures in patients with dementia. Geriatr Orthop Surg Rehabil 1(2):63-72

3. Dyer S et al (2017) Rehabilitation following hip fracture. In: Falaschi P, Marsh D (eds) Orthogeriatrics. Springer, Switzerland, pp 145-163

4. National Clinical Guideline Centre (2011) The Management of Hip Fracture in Adults. National Clinical Guideline Centre, London. www.ncgc.ac.uk

5. Olsson L-E et al (2007) Effects of nursing interventions within and integrated care pathway for patients with hip fracture. J Adv Nurs 58(2):116-125

6. Curtis L (2009) Unit costs of health \& social care 2009. Personal Social Services Research Unit, Canterbury, Kent (Guideline Ref ID: CURTIS2009)

7. Sherrington C, Whitney JC, Lord SR, Herbert RD, Cumming RG, Close JC (2008) Effective exercise for the prevention of falls: a systematic review and meta-analysis. J Am Geriatr Soc 56(12):2234-2243

8. Gesar B (2017) Older patients' perception of their own capacity to regain pre-fracture function after hip fracture surgery - an explorative qualitative study. Int J Orthop Trauma Nurs 24:50-58

9. Crotty M et al (2010) Rehabilitation interventions for improving physical and psychosocial functioning after hip fracture in older people (review) 5 Copyright @ 92010 The Cochrane Collaboration. Wiley

10. Maclean N, Pound P (2000) A critical review of the concept of patient motivation in the literature on physical rehabilitation. Soc Sci Med 50:495-506

11. Hitch B et al (2016) Evaluation of a team-based, transition-of-care management service on 30-day readmission rates. NC Med J 77(2):87-92

12. Gunadi $S$ et al (2015) Development of a collaborative transitions-of-care program for heart failure patients. Am J Health Syst Pharm 72(13):1147-1152

13. Bauer M et al (2009) Hospital discharge planning for frail older people and their family. Are we delivering best practice? A review of the evidence. J Clin Nurs 18(18):2539-2546

14. Kripalani $S$ et al (2007) Deficits in communication and information transfer between hospitalbased and primary care physicians: implications for patient safety and continuity of care. JAMA 297:831

15. National Patient Safety Foundation/Institute for Healthcare Improvement (nd) Ask Me 3. http://www.npsf.org/?page=askme3

16. Blaylock A, Cason CL (1992) Discharge planning predicting patients' needs. J Gerontol Nurs 18:5-10

17. Randmaa $M$ et al (2014) SBAR improves communication and safety climate and decreases incident reports due to communication errors in an anaesthetic clinic: a prospective intervention study. BMJ Open 4:e004268. https://doi.org/10.1136/bmjopen-2013-004268

18. Holland D, Harris M (2007) Discharge planning, transitional care, coordination of care, and continuity of care: clarifying concepts and terms from the hospital perspective. Home Health Care Serv Quart 26(4):3-19

19. Kangovi $\mathrm{S}$ et al (2014) The use of participatory action research to design a patient-centered community health worker care transitions intervention. Healthc (Amst) 2(2):136-144

20. Gulliford M et al (2006) What is 'continuity of care'? J Health Serv Res Policy 11(4):248-250

21. Cohen EL, Cesta TG (1993) Nursing case management: from concept to evaluation. Mosby, St Louis 
Open Access This chapter is licensed under the terms of the Creative Commons Attribution 4.0 International License (http://creativecommons.org/licenses/by/4.0/), which permits use, sharing, adaptation, distribution and reproduction in any medium or format, as long as you give appropriate credit to the original author(s) and the source, provide a link to the Creative Commons license and indicate if changes were made.

The images or other third party material in this chapter are included in the chapter's Creative Commons license, unless indicated otherwise in a credit line to the material. If material is not included in the chapter's Creative Commons license and your intended use is not permitted by statutory regulation or exceeds the permitted use, you will need to obtain permission directly from the copyright holder. 